\title{
Análise da dose vocal em pessoas cisgênero: resultados preliminares
}

\section{Analysis of vocal dose in cisgender people: preliminary results}

\author{
Caroline de Lima' (1), Alana Dantas Barros² (i), Ana Cristina Côrtes Gama³ (1)
}

\section{RESUMO}

Objetivo: Verificar a diferença das medidas de frequência fundamental, intensidade e de dose vocal entre mulheres e homens cisgêneros, em situações de mesma demanda de voz. Métodos: Trata-se de uma pesquisa observacional transversal. Participaram do Grupo 1 cinco homens cisgêneros, com idade entre 21 e 24 anos, e do Grupo 2, cinco mulheres cisgêneras, com idade entre 22 e 25 anos, todos sem queixas vocais e estudantes do curso de Fonoaudiologia. Os indivíduos foram pareados por demanda vocal, sendo que todos estavam matriculados nas mesmas disciplinas do curso de graduação. Todos os participantes se autodefiniram como cisgêneros, ou seja, identificaram-se com o sexo designado ao nascer. A coleta foi realizada de forma simultânea aos pares (um participante do G1 e um do G2), por um período contínuo de dez horas. Para a coleta de dados, utilizaram-se dois dosímetros da marca VoxLog ${ }^{\circledR}$. Para comparação das medidas entre os grupos utilizou-se o teste t de Student, com nível de confiança de $95 \%$. Resultados: Observou-se que as mulheres cisgêneras apresentaram maiores valores de frequência fundamental $(\mathrm{p}=0,001)$, porcentagem de fonação $(\mathrm{p}=0,037)$, dose cíclica $(\mathrm{p}=0,002)$ e dose de distância $(\mathrm{p}=0,008)$. A intensidade da voz de ambos os grupos foi semelhante no período avaliado. Conclusão: Mulheres cisgêneras apresentam maiores valores de frequência fundamental, porcentagem de fonação, dose cíclica e dose de distância, do que homens cisgêneros. Avaliar a dose vocal entre os gêneros é importante para a melhor compreensão dos fatores etiológicos das disfonias comportamentais e para definir uma reabilitação vocal mais personalizada.

Palavras-chave: Voz; Identidade de gênero; Disfonia; Dosimetria; Fonoaudiologia

\begin{abstract}
Purpose: The purpose of this study is to verify the difference in fundamental frequency, intensity and vocal dose measurements between male and female cisgender people, in same voice demand situations. Methods: This is a cross-sectional observational case study. Group 1 participated with five male people, aged between 21 and 24 years old and Group 2 with five female people, aged between 22 and 25 years old, both without vocal complaints, students of the Speech-language pathology course. The individuals were paired by vocal demand, and all were enrolled in the same activists of the undergraduate course. All participants defined themselves as cisgender, that is, they identified themselves with the gender designated at birth. The data was carried out at the same time, for a period of 10 hours, while the participants carried out their daily activities for that period. For data collection, two dosimeters of VoxLog ${ }^{\circledR}$, were used. Student's t test was used to compare measures between groups. The $95 \%$ confidence level was considered. Results: It was observed that women have higher values of fundamental frequency $(\mathrm{p}=0,001)$, percentage of phonation $(\mathrm{p}=0,037)$, cyclic dose $(\mathrm{p}=0,002)$ and distance dose $(\mathrm{p}=0,008)$. The voice intensity of both groups was similar in the period evaluated. Conclusion: cisgender women have higher values of fundamental frequency, percentage of phonation, cyclic dose and distance dose than cisgender men. Assessing the vocal dose between genders is important for a better understanding of the etiological factors of behavioral dysphonia and to define a more personalized vocal rehabilitation.
\end{abstract}

Keywords: Voice; Gender identity; Dysphonia; Dosimetry; Speech, Language and Hearing Sciences

Trabalho realizado no Curso de Fonoaudiologia, Universidade Federal de Minas Gerais - UFMG - Belo Horizonte (MG), Brasil.

${ }^{1}$ Curso de graduação em Fonoaudiologia, Faculdade de Medicina, Universidade Federal de Minas Gerais - UFMG - Belo Horizonte (MG), Brasil.

${ }^{2}$ Programa de Pós-graduação em Saúde Coletiva (doutorado), Faculdade de Ciências da Saúde, Universidade de Brasília - UnB - Brasília (DF), Brasil.

${ }^{3}$ Departamento de Fonoaudiologia, Faculdade de Medicina, Universidade Federal de Minas Gerais - UFMG - Belo Horizonte (MG), Brasil.

Conflito de interesses: Não.

Contribuição dos autores: CL coletou e analisou os dados e organizou o texto; ADB e ACCG revisaram criticamente o conteúdo do manuscrito.

Financiamento: Conselho Nacional de Desenvolvimento Científico e Tecnológico-Brasil (CNPq) (nº 309108/2019-5).

Autor correspondente: Ana Cristina Côrtes Gama. E-mail: anaccgama@gmail.com

Recebido: Abril 06, 2021; Aceito: Junho 17, 2021 


\section{INTRODUÇÃO}

O conceito de gênero é formado a partir de uma construção social, com base em diferentes aspectos, sejam relacionais ou culturais, e que se cerca de diferentes valores, identidades, comportamentos e sentimentos ${ }^{(1)}$. É diferente do conceito de sexo, que classifica os indivíduos como homens ou mulheres, segundo as características anatômicas ${ }^{(1)}$.

$\mathrm{O}$ sexo determina algumas características laríngeas ${ }^{(2,3)} \mathrm{e}$ aerodinâmicas ${ }^{(4)}$ que influenciam diferenças no padrão fonatório de pessoas do sexo masculino e feminino.

Em relação à anatomia laríngea, as pregas vocais (PPVV) de pessoas do sexo masculino são maiores e mais largas do que as do sexo feminino, e a posição vertical da laringe é mais baixa no sexo masculino ${ }^{(2)}$. A produção vocal também sofre influência das estruturas faciais, como a forma, o tamanho, a densidade e a tensão, que são diferentes entre os $\operatorname{sexos}^{(2)}$.

$\mathrm{O}$ ácido hialurônico encontrado na lâmina própria das PPVV é descrito como uma substância que interfere nos padrões vibratórios da túnica mucosa ${ }^{(3)}$. Suas propriedades parecem diminuir a chance de se desenvolver lesão de massa por impacto durante a fonação, devido a sua importante função na absorção do fonotrauma ${ }^{(3)}$, e as pessoas do sexo masculino parecem apresentar maior quantidade desta substância nas PPVV que as do sexo feminino ${ }^{(3)}$.

Para falantes do português brasileiro, a frequência fundamental média para adultos do sexo masculino é $113 \mathrm{~Hz}$ e para adultas do sexo feminino, $204 \mathrm{~Hz}$ (média de 18 a 45 anos de idade) ${ }^{(4)}$. $\mathrm{O}$ tempo máximo de fonação (TMF) esperado para adultos do sexo feminino é de acima de 14 segundos e do sexo masculino, acima de 20 segundos $^{(4)}$. A capacidade vital (CV) também se difere entre os sexos. A literatura mostra que os valores mínimos para homens são de $2.200 \mathrm{ml}$ e para mulheres, de $2.100 \mathrm{ml}^{(4)}$.

Como exposto, existem muitas diferenças na voz que são dependentes de características biológicas determinadas pelo sexo do falante, mas a questão do gênero também interfere nos padrões comunicativos.

As pesquisas mostram que existem perfis relacionados à personalidade e comportamentos que são mais femininos ou masculinos. Os homens parecem apresentar maior engajamento em atividades físicas coletivas e de caráter competitivo; já as mulheres, em atividades individuais, que requerem do corpo menos força física ${ }^{(5)}$. Mulheres apresentaram maiores escores de estresse, ansiedade e depressão que os homens ${ }^{(6)}$. Em outro estudo, homens apresentaram maiores pontuações no traço de personalidade de abertura à mudança; já as mulheres apresentaram maiores pontuações no traço de personalidade de amabilidade ${ }^{(7)}$.

Com relação às mulheres, estudo apontou que elas possuem maior quantidade de fala em ambiente de trabalho, quando comparadas aos homens ${ }^{(8)}$. Outro estudo mostrou que mulheres possuem maior interesse e procura sobre orientações com relação à saúde vocal e que os homens denotam maior desconhecimento sobre higiene $\operatorname{vocal}^{(9)}$.

Diferenças na voz estão, portanto, relacionadas a questões que envolvem o gênero, como aspectos dos padrões comunicativos, do comportamento da voz e, provavelmente, dos parâmetros de dose vocal.

A dose da voz é definida como a exposição do tecido da prega vocal (PV) à vibração ao longo do tempo ${ }^{(10)}$. O dosímetro vocal é um equipamento portátil usado para se obter importantes parâmetros do comportamento vocal durante atividades normais do dia. O objetivo desse equipamento é medir a intensidade da voz em termos de nível de pressão sonora (dBNPS), a frequência fundamental $(\mathrm{Hz})$ e a duração da atividade vocal dos participantes, definida pelo tempo de uso de voz. A partir desses três parâmetros vocais, os valores de dose da voz são mensurados ${ }^{(10)}$. Esses valores podem ser usados como dados de avaliação complementar para auxiliar na análise da complexa relação entre o uso da voz, fadiga vocal e tempo de recuperação vocal $^{(10)}$.

A literatura sugere que, em geral, os falantes do sexo feminino apresentam dose cíclica mais alta, quando comparadas aos falantes do sexo masculino, pois essa dose é sensível à média da frequência fundamental ${ }^{(11)}$. Além disso, o comportamento vocal entre pessoas de gêneros distintos se difere com relação às exigências vocais sociais e/ou profissionais e acredita-se que a dose vocal também seja sensível a essas diferentes demandas relacionadas ao gênero.

A literatura denomina as pessoas cisgêneros, ou "cis", aquelas que se identificam com o gênero atribuído no nascimento, e as pessoas "trans", aquelas que não se identificam com o gênero determinado ao nascimento ${ }^{(12)}$. Esses termos, apesar de comuns na literatura, não são consensuais, devido à própria variedade de experiências humanas sobre como se identificar a partir de seu corpo ${ }^{(12)}$.

No caso das pessoas "trans" e com variabilidade de gênero, a voz pode ser um fator marcante na percepção do gênero e a não conformidade entre eles pode gerar sentimentos de inadequação e consequente redução da comunicação e da expressão por meio da $\operatorname{voz}^{(13,14)}$.

É importante compreender as questões do gênero na dose vocal para se definir protocolos mais personalizados de tratamento para as disfonias, principalmente as de base comportamental. A literatura evidencia maior prevalência de disfonia no sexo feminino ${ }^{(15)}$ e o comportamento vocal, influenciado pelo gênero, parece ser um importante fator etiológico das alterações vocais ${ }^{(16)}$. Analisar o comportamento da dose vocal entre os gêneros é importante para a melhor compreensão dos fatores etiológicos das disfonias comportamentais e para se definir processos de reabilitação vocal mais individualizados.

Diante do exposto, o objetivo desta pesquisa foi levantar resultados preliminares sobre a diferença das medidas de frequência fundamental, intensidade e de dose vocal entre pessoas que se autodefiniram como cisgênero em situações de mesma demanda de voz.

\section{MÉTODO}

Trata-se de um estudo piloto do tipo observacional analítico transversal, aprovado pelo Comitê de Ética em Pesquisa da Universidade Federal de Minas Gerais -UFMG, sob o parecer 3.059.941. Todos os participantes leram e assinaram o Termo de Consentimento Livre e Esclarecido (TCLE).

Foram selecionados dois grupos para a pesquisa: o primeiro grupo (G1) foi constituído por cinco pessoas do sexo masculino sem queixas vocais, com idade entre 21 e 24 anos (média $=22,2$ anos), e o segundo grupo (G2), constituído por cinco pessoas do sexo feminino sem queixa de voz, com idade entre 22 e 25 anos (média $=23,2$ anos). Não houve diferença de idade entre os grupos $(\mathrm{p}=0,322)$. Todos os participantes da pesquisa eram estudantes do curso de Fonoaudiologia da UFMG. 
Foram critérios de inclusão ter idade entre 18 e 45 anos, por ser o período de maior estabilidade vocal; ausência de queixa vocal; na avaliação fonoaudiológica, apresentar qualidade vocal neutra e se autodefinir como pessoa cisgênero.

Para a avaliação da ausência de sintomas vocais, os participantes foram questionados sobre a presença de fadiga da voz e/ou de desconforto fonatório. Foram incluídos os participantes com ausência de sintomas de fadiga e de desconforto vocal.

Para a análise da qualidade vocal, foi realizada avaliação perceptivo-auditiva da voz por duas fonoaudiólogas com experiência de mais de cinco anos em avaliação vocal. A análise foi realizada de forma consensual. Foi avaliado o grau geral da disfonia (G), na tarefa de vogal /a/ sustentada de forma habitual e de fala espontânea, e classificado em uma escala de 4 pontos, sendo zero (0) alteração ausente e três (3), alteração de grau intenso. Foram incluídos os participantes com qualidade vocal neutra (G0).

Para a autodefinição de pessoa cisgênero, foi perguntado aos participantes se eles se identificavam com o gênero que lhes foi atribuído ao nascimento. Foram definidas como cisgêneros as pessoas que responderam positivamente.

Como critérios de exclusão, para os dois grupos, foram considerados indivíduos que referiram estar em período alérgico ou gripal. Para o grupo de mulheres cisgêneras (G2), foram excluídas grávidas e aquelas que se encontravam no período pré-menstrual ou menstrual.

Os indivíduos de ambos os grupos (G1 e G2) foram pareados por demanda vocal, sendo que todos estavam matriculados no mesmo período e nas mesmas disciplinas do curso de graduação em Fonoaudiologia da UFMG.

A coleta foi realizada simultaneamente em pares de indivíduos (um do G1 e um do G2), por um período de dez horas contínuas, enquanto os participantes realizavam suas atividades. Portanto, durante a coleta, cada par de indivíduos estava executando as mesmas atividades universitárias.

A coleta de dados ocorreu na Faculdade de Medicina da UFMG, aos pares, e no período contínuo de dez horas cada indivíduo registrou em um quadro controle informações referentes às atividades realizadas durante o dia. Os participantes deveriam marcar se as atividades acadêmicas eram práticas (observação de atendimentos clínicos), teóricas ou de estágios clínicos (atendimento de pacientes e posterior supervisão com o docente responsável pela disciplina). Ambos os grupos realizaram, aos pares, exatamente as mesmas atividades letivas durante todo o período de gravação.

Para a coleta de dados, foram utilizados dois dosímetros da marca VoxLog®, da Sonvox, modelo 3.1, compostos por um microfone, um acelerômetro e uma unidade portátil que armazenou os dados fonatórios. Um dosímetro foi utilizado por um participante do G1 e o outro, por um participante do G2. As gravações foram simultâneas (Figura 1).

$\mathrm{O}$ acelerômetro foi fixado por fita micropore no pescoço do participante, próximo à cartilagem tireóidea e à unidade portátil na cintura. Os dados coletados foram compostos pelos seguintes parâmetros ${ }^{(17)}$ :

1. Frequência fundamental (f) : é o número de ondas sonoras por unidade de tempo e é medida em Hz (Hertz);

2. Intensidade vocal: representa a quantidade de energia do som produzido, medida em dBNPS (Decibel nível de pressão sonora);
3. Porcentagem de fonação: comparada com o tempo decorrido no período monitorado;

4. Dose cíclica (Dc): quantifica o número de oscilações das PPVV durante o período gravado. É calculada a partir do tempo de fonação e da média da frequência fundamental. Medida em milhares de ciclos;

5. Dose da distância (Dd): é a distância total percorrida pelo tecido das PPVV na trajetória cíclica durante a vibração e depende não somente do tempo total de fonação e frequência fundamental, mas da amplitude da vibração da PV. Medida em metros.

Os dados coletados foram analisados no computador, por meio de software específico do equipamento. Como a dose de distância não é calculada pelo software disponível, foi definida a partir dos cálculos propostos na literatura ${ }^{(17)}$

A análise estatística dos dados foi realizada por meio do programa estatístico MINITAB, versão 17. Primeiramente, realizou-se uma análise descritiva dos dados, com medidas de tendência central e dispersão. Posteriormente, foi utilizado o teste de Anderson-Darling para verificar a normalidade da amostra. Para comparação das medidas entre os grupos, utilizouse o teste paramétrico t de Student. Considerou-se o nível de confiança de $95 \%$.

\section{RESULTADOS}

As mulheres cisgêneras apresentaram maiores valores de frequência fundamental, porcentagem de fonação, de dose cíclica e de distância. $\mathrm{O}$ único parâmetro acústico que não se diferenciou entre os grupos foi o da intensidade da voz (Tabela 1).

\section{DISCUSSÃO}

A frequência fundamental foi maior no grupo feminino cisgênero. Os fatores que podem explicar tais resultados estão relacionados ao sexo, como evidenciado pela literatura ${ }^{(18)}$, pelo fato de as pessoas do sexo feminino apresentarem PPVV menores $^{(2)}$ e com trato vocal mais encurtado ${ }^{(3)}$. Apesar de a

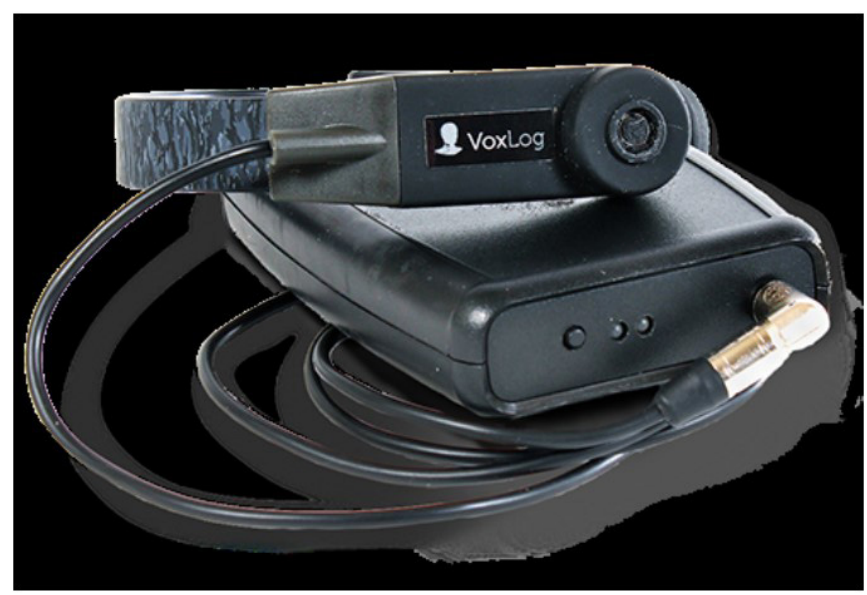

Figura 1. Dosímetro vocal da marca VoxLog® da Sonvox modelo 3.1 
Tabela 1. Comparação dos parâmetros vocais entre os gêneros masculino e feminino

\begin{tabular}{|c|c|c|c|c|c|c|c|c|c|}
\hline \multirow{2}{*}{ Parâmetro } & \multicolumn{4}{|c|}{ Gênero masculino } & \multicolumn{4}{|c|}{ Gênero Feminino } & \multirow{2}{*}{$\begin{array}{l}\text { Valor } \\
\text { de } p\end{array}$} \\
\hline & Média & DP & Min & Máx & Média & DP & Mín & Máx & \\
\hline Frequência fundamental $(\mathrm{Hz})$ & 169,8 & 35,41 & 121,53 & 204,67 & 286,55 & 16,28 & 267,52 & 303,56 & $0,001^{*}$ \\
\hline Intensidade vocal (dBNPS) & 86,56 & 1,98 & 84,85 & 89,63 & 87,61 & 0,909 & 86,26 & 88,75 & 0,331 \\
\hline Porcentagem de fonação (\%) & 9,632 & 2,55 & 6,32 & 12,42 & 13,662 & 2,423 & 10,15 & 16,03 & $0,037^{*}$ \\
\hline Dose cíclica (milhares de ciclos) & 445 & 0,391 & 319 & 553 & 1164,2 & 244,5 & 804 & 1406 & $0,002^{*}$ \\
\hline Dose de distância (m) & 3940,7 & 1000,2 & 25559,9 & 5325,8 & 6472,4 & 1181,4 & 4593,8 & 7686,5 & $0,008^{*}$ \\
\hline
\end{tabular}

*Teste $t$ significativo a $5 \%$

Legenda: $\mathrm{Hz}$ = Hertz; dBNPS = decibel nível de pressão sonora; $\mathrm{m}$ = metro; $\mathrm{DP}$ = desvio padrão; Min = valor mínimo; Máx = valor máximo

literatura mostrar resultados referentes à $\mathrm{f}_{0}$ acústica, sabe-se que os valores de frequência fundamental estimados pelo microfone e pelo acelerômetro apresentam correlação perfeita ${ }^{(19)}$, o que permite analisar os dados de $\mathrm{f}_{0}$ acústica apontados pela literatura com os valores de $\mathrm{f}_{0}$ obtidos neste estudo. Não foram observados estudos que tenham analisado valores de $\mathrm{f}_{0}$ relacionados ao gênero dos falantes.

No Brasil, autores referenciam a faixa de normalidade da $\mathrm{f}_{0}$ como sendo entre 80 e $150 \mathrm{~Hz}$ para o sexo masculino e de 150 a $250 \mathrm{~Hz}$ para o sexo feminino ${ }^{(3)}$. Os valores de $\mathrm{f}_{0}$ desta pesquisa foram maiores do que os relatados pela literatura e esse fato pode ser decorrente da interferência da intensidade da voz e do aquecimento vocal ao longo da coleta dos dados pelo dosímetro de voz.

Já foi evidenciado que os valores de $\mathrm{f}_{0}$ aumentam quando a intensidade aumenta ${ }^{(20)}$ e que há uma elevação da $\mathrm{f}_{0}$ com o aquecimento vocal decorrente do uso contínuo da voz ao longo do dia ${ }^{(21)}$. Como o período de coleta foi de dez horas, o aumento da $\mathrm{f}_{0}$ pode ter sido ocasionado pelo uso da voz durante o longo período da coleta ${ }^{(21)}$ e pelo fato de os participantes estarem, em alguns momentos do dia, em ambientes abertos e com ruído, o que pode ter elevado os valores de intensidade e, consequentemente, da $\mathrm{f}_{0}^{(20)}$.

A intensidade é uma medida física da quantidade de energia do som medida em $\mathrm{dBNPS}^{(10)}$. No presente estudo, a média da intensidade não se diferiu entre os grupos e os valores médios da intensidade observados foram maiores que os valores de intensidade habituais descritos na literatura ${ }^{(22)}$.

Não foram encontradas pesquisas que tenham comparado esse parâmetro acústico entre os gêneros e os sexos dos falantes, e o resultado da intensidade, medida pelo dosímetro vocal, apresentar-se maior do que a literatura evidencia ${ }^{(22)}$, o que pode ser justificado pelo fato de os participantes estarem expostos a ambientes de comunicação abertos e com ruído. A literatura mostra que a intensidade é dependente da situação do discurso ${ }^{(23)}$ e da presença de alteração vocal(24). É importante salientar que, como os participantes dos dois grupos foram expostos aos pares às mesmas situações comunicativas durante a coleta, o ambiente e o nível de ruído foram variáveis que interferiram de forma similar nos valores de ambos os grupos.

Os resultados do parâmetro de intensidade desta pesquisa podem estar relacionados às mesmas condições ambientais de fala dos participantes de ambos os grupos ${ }^{(23,25)}$ e por estes não apresentarem nenhum tipo de alteração vocal ${ }^{(24)}$.

Pesquisas que avaliaram a interferência do gênero na dose vocal dos falantes são escassas, o que limitou a comparação dos achados desta pesquisa com dados da literatura.

A porcentagem de fonação é uma medida de dose que avalia o quanto a PV vibrou, quantificando o tempo que a pessoa usou a voz ao longo das atividades de comunicação ${ }^{(17)}$, sendo medida em porcentagem. Das medidas de dose vocal que foram avaliadas, a porcentagem de fonação é o único parâmetro que não é dependente da $\mathrm{f}_{0}$.

Os resultados demonstraram que as mulheres cisgêneras falaram em torno de $14 \%$ do tempo em que foram monitoradas e os homens cisgêneros, que estavam submetidos à mesma situação de ambiente comunicativo e executando as mesmas atividades ao longo do dia, tiveram porcentagem de fonação em torno de $11 \%$, sugerindo que a mulheres cisgêneras usaram mais a voz ao longo do dia.

Esse resultado reafirma a literatura, que evidenciou maiores valores de porcentagem de fonação para pessoas do sexo feminino, tanto nas atividades ocupacionais (30,7\%), quanto nas não ocupacionais (14,7\%), quando comparadas às pessoas do sexo masculino $(27,4 \% \text { e } 13,7 \%)^{(26)}$. Outra pesquisa, realizada com um gravador ativado eletronicamente (EAR), demonstrou que o sexo feminino tende a usar cerca de $7 \%$ mais palavras em um dia do que o sexo masculino ${ }^{(27)}$. Não foram encontrados estudos que tenham comparado os valores de porcentagem de fonação entre os gêneros.

Para o grupo de transgêneros, também não se observaram pesquisas relacionadas à dose vocal. Um estudo mostrou que pessoas "trans" têm apresentado baixa qualidade de vida relacionada à voz ${ }^{(28)}$ e que homens "trans" têm relatado sensação de fadiga vocal e dificuldade de projeção da voz em situações laborais como professores ${ }^{(29)}$. Tais resultados podem ser justificados por fatores relacionados à qualidade vocal de pessoas "trans". Pesquisas relacionadas à dose vocal de "trans" são importantes para a melhor compreensão da demanda da voz na gênese de tais sintomas.

As doses cíclica e de distância apresentaram maiores valores no grupo de mulheres cisgêneras. Essas duas medidas dependem do valor da $\mathrm{f}_{0} \mathrm{e}$, como esta é maior no sexo feminino, os resultados observados podem ter sido influenciados por esse parâmetro acústico.

A literatura não apresenta estudos comparando os valores de dose vocal entre o gênero e o sexo dos falantes. Em outras populações, os valores de dose vocal variam de acordo com as atividades realizadas, como, por exemplo, uso de amplificação sonora em professores ${ }^{(24)}$, atividades de canto $^{(27)}$ e tarefas de sobrecarga vocal em adultos sem alteração laríngea ${ }^{(30)}$. Apesar de ambas as doses utilizarem em seus cálculos os dados da $\mathrm{f}_{0}$, é lícito supor que, como a porcentagem de fonação apresentou maiores valores no grupo de mulheres cisgêneras, esse resultado pode também estar associado ao maior uso da voz no gênero feminino durante as atividades realizadas ao longo do dia.

Como limitações desta pesquisa, se destaca o número reduzido de participantes, o que limita a validade externa dos resultados. Pesquisas futuras com maior número de indivíduos 
são importantes para ampliar os resultados preliminares que foram obtidos nesta pesquisa.

\section{CONCLUSÃO}

Mulheres cisgêneras apresentam maiores valores de frequência fundamental, porcentagem de fonação, dose cíclica e dose de distância, que homens cisgêneros. A intensidade da voz de ambos os gêneros foi semelhante no período de uso vocal avaliado.

\section{AGRADECIMENTOS}

Conselho Nacional de Desenvolvimento Científico e Tecnológico-Brasil (CNPq) (nº 309108/2019-5).

\section{REFERÊNCIAS}

1. Praun AG. Sexualidade, gênero e suas relações de poder. Revista Húmus. 2001;1:55-65.

2. Butler JE, Hammond TH, Gray SD. Gender-related differences of hyaluronic acid distribution in the human vocal fold. Laryngoscope. 2001;111(5):907-11. http://dx.doi.org/10.1097/00005537-20010500000029. PMid:11359176.

3. Chan RW, Gray SD, Titze IR. The importance of hyaluronic acid in vocal fold biomechanics. Otolaryngol Head Neck Surg. 2001;124(6):607-14. http://dx.doi.org/10.1177/019459980112400602. PMid:11391249.

4. Behlau MS, Madazio G, Pontes P. Disfonias organofuncionais. In: Behlau MS, organizador. Voz: o livro do especialista. Rio de Janeiro: Revinter; 2001. p. 295-341.

5. Salles-Costa R, Heilborn ML, Werneck GL, Faerstein E, Lopes CS. Gênero e prática de atividade física de lazer. Cad Saude Publica. 2003;19(2, Suppl 2):S325-33. http://dx.doi.org/10.1590/S0102311X2003000800014. PMid:15029352.

6. Dietrich M, Verdolini Abbott K, Gartner-Schmidt J, Rosen CA. The frequency of perceived stress, anxiety, and depression in patients with common pathologies affecting voice. J Voice. 2008;22(4):472-88. http://dx.doi.org/10.1016/j.jvoice.2006.08.007. PMid:18395419.

7. Gonçalves MP, Gouveia VV, Cavalcanti TM, Bezerra CC, Medeiros ED, Oliveira GF, et al. Atributos desejáveis de parceiro ideal: podem variar segundo o sexo e o lugar de residência? Temas Psicol. 2018;26(3):1221-34.

8. Padilha MP, Moreti F, Raize T, Sauda C, Lourenço L, Oliveira GI, et al. Grau de quantidade de fala e intensidade vocal de teleoperadores em ambiente laboral e extralaboral. Rev Soc Bras Fonoaudiol. 2012;17(4):38590. http://dx.doi.org/10.1590/S1516-80342012000400004.

9. Zimmer V, Cielo CA, Ferreira FM. Comportamento vocal de cantores populares. Rev CEFAC. 2012;14(2):298-307. http://dx.doi.org/10.1590/ S1516-18462011005000101.

10. Svec JG, Titze IR, Popolo PS. Estimation of sound pressure levels of voiced speech from skin vibration of the neck. J Acoust Soc Am. 2005;117(3 Pt 1):1386-94. http://dx.doi.org/10.1121/1.1850074. PMid: 15807026 .

11. Gaskill CS, Cowgill JG, Tinter SR. Vocal dosimetry: a graduate level voice pedagogy course experience. J Sing. 2013;69(5):543.
12. Jesus JG. Orientações sobre identidade de gênero: conceitos e termos: guia técnico sobre pessoas transexuais, travestis e demais transgêneros, para formadores de opinião [Internet]. Brasília; 2012 [citado em 2021 Abr 5]. 42 p. Disponível em: http://www.diversidadesexual.com.br/ wp-content/uploads/2013/04/G\%C3\%8ANERO-CONCEITOS-ETERMOS.pdf

13. Hancock A, Haskin G. Speech-Language Pathologists' Knowledge and Attitudes Regarding Lesbian, Gay, Bisexual, Transgender, and Queer (LGBTQ) Populations. Am J Speech Lang Pathol. 2015;24(2):206-21. http://dx.doi.org/10.1044/2015_AJSLP-14-0095. PMid:25654222.

14. Azul D, Hancock AB, Nygren U. Forces affecting voice function in gender diverse people assigned female at birth. J Voice. 2020;35(4):662. e15-e34. http://dx.doi.org/10.1016/j.jvoice.2020.01.001.

15. Roy N, Merrill RM, Thibeault S, Parsa RA, Gray SD, Smith EM. Prevalence of voice disorders in teachers and the general population. J Speech Lang Hear Res. 2004;47(4):281-93. http://dx.doi.org/10.1044/10924388(2004/023). PMid:15157130.

16. Ohlsson AC, Andersson EM, Södersten M, Simberg S, Barregård L. Prevalence of voice symptoms and risk factors in teacher students. J Voice. 2012;26(5):629-34. http://dx.doi.org/10.1016/j.jvoice.2011.11.002. PMid:22578438.

17. Titze IR, Svec JG, Popolo PS. Vocal dose measures: quantifying accumulated vibration exposure in vocal fold tissues. J Speech Lang Hear Res. 2003;46(4):919-32. http://dx.doi.org/10.1044/10924388(2003/072). PMid:12959470.

18. Mifune E, Justino VSS, Camarg Z, Gregio F. Análise acústica da voZ do idoso: caracterização da frequência fundamental. Rev CEFAC. 2007;9(2):238-47. http://dx.doi.org/10.1590/S1516-18462007000200013.

19. Oliveira RC, Gama ACC, Magalhães MDC. Fundamental voice frequency: acoustic, electroglottographic, and accelerometer measurement in individuals with and without vocal alteration. J Voice. 2021;35(2):17480. http://dx.doi.org/10.1016/j.jvoice.2019.08.004. PMid:31575435.

20. Rabelo ATV, Santos JN, Souza BO, Gama ACC, de Castro Magalhães $\mathrm{M}$. The influence of noise on the vocal dose in women. J Voice. 2019;33(2):214-9. http://dx.doi.org/10.1016/j.jvoice.2017.10.025. PMid:29290547.

21. Hunter EJ, Titze IR. Variations in intensity, fundamental frequency and voicing for teachers in occupational versus nonoccupational settings. J Speech Lang Hear Res. 2010;53(4):862-75. http://dx.doi. org/10.1044/1092-4388(2009/09-0040). PMid:20689046.

22. Koishi HU, Tsuji DH, Imamura R, Sennes LU. Variação da intensidade vocal: estudo da vibração das pregas vocais em seres humanos com videoquimografia. Rev Bras Otorrinolaringol (Engl Ed). 2003;69(4):46470. http://dx.doi.org/10.1590/S0034-72992003000400005.

23. Remacle A, Morsomme D, Finck C. Comparison of vocal loading parameters in kindergarten and elementary school teachers. J Speech Lang Hear Res. 2014;57(2):406-15. http://dx.doi.org/10.1044/2013 JSLHR-S-12-0351. PMid:24129011.

24. Gaskill CS, O’Brien SG, Tinter SR. The effect of voice amplification on occupational vocal dose in elementary school teachers. J Voice. 2012;26(5):667.e19-27. http://dx.doi.org/10.1016/j.jvoice.2011.10.010. PMid:22521533.

25. Gramming P, Sundberg J, Ternstrom S, Leanderson R, Perkins W. Relationship between changes in voice pitch and loudness. J Voice. 1988;2(2):118-26. http://dx.doi.org/10.1016/S0892-1997(88)80067-5.

26. Mehl MR, Vazire S, Ramirez-Esparza N, Slatcher RB, Pennebaker JW. Are women really more talkative than men? Science. 2007;317(6):82. http://dx.doi.org/10.1126/science.1139940. PMid:17615349. 
27. Schloneger MJ, Hunter EJ. Assessments of voice use and voice quality among college/university singing students ages 18-24 through ambulatory monitoring with a full accelerometer signal. J Voice. 2017;31(1):124.e21-30. http://dx.doi.org/10.1016/j.jvoice.2015.12.018. PMid:26897545.

28. Dornelas R, Guedes-Granzotti RB, Souza AS, Jesus AKB, Silva K. Quality of life and voice: the vocal self-perception of transgender people. ACR. 2020;25:e2196.
29. Barros DB, Cavadinha ET, Mendonça AVM. A percepção de homens trans sobre a relação entre voz e expressão de gênero em suas interações sociais. Tempus Actas de Saúde Colet. 2018;11(4):9-24.

30. Echternach M, Nusseck M, Dippold S, Spahn C, Richter B. Fundamental frequency, sound pressure level and vocal dose of a vocal loading test in comparison to a real teaching situation. Eur Arch Otorhinolaryngol. 2014;271(12):3263-8. http://dx.doi.org/10.1007/s00405-014-3200-6. PMid:25012705. 